Geografia e Ordenamento do Território, Revista Electrónica

Centro de Estudos de Geografia e Ordenamento do Território

http://cegot.org

ISSN :2182-1267

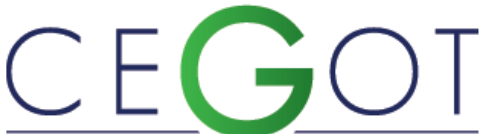

Centro de Estudos de Geografia e Ordenamento do Território
DAVID, NUNO

Universidade de Lisboa, Instituto Superior Técnico, Departamento de Engenharia Civil e Arquitetura, CERIS

Av. Rovisco Pais 1049-001, Lisboa, Portugal

nunomigueldavid@gmail.com

\title{
O valor económico da paisagem - uma análise comparativa entre métodos de valoração económica
}

\author{
Landscape economic value - a comparative analysis through economic \\ valuation methods
}

Referência: David, Nuno (2017). O valor económico da paisagem - uma análise comparativa entre métodos de valoração económica. Revista de Geografia e Ordenamento do Território (GOT), n. 12 (dezembro). Centro de Estudos de Geografia e Ordenamento do Território, p. 101-125, dx.doi.org/10.17127/got/2017.12.005

\section{RESUMO}

O presente trabalho discute a importância em atribuir valor económico à paisagem, nomeadamente quando este é expresso em escala monetária e quando decorre em contexto de mercado ou de ausência de mercado. Com base na literatura de referência, procede-se a uma análise comparativa de métodos de valoração económica para paisagens e ecossistemas. No final discutem-se os desafios que se podem colocar à administração pública, quanto à captura e internalização no processo de planeamento, do valor que decorre de benefícios e externalidades provenientes de paisagens e ecossistemas.

Palavras-chave: Paisagem, valor económico, serviços de ecossistemas, benefícios.

\section{ABSTRACT}

This paper discusses the importance of assigning economic value to the landscape, in particular when it is expressed in monetary scale and when held in market context or market absence. Based on literature, a comparative analysis of economic valuation methods for landscapes and ecosystems is carried out. At the end, we discuss the challenges that may arise to public administration, regarding the capture and internalization of values in to the 
planning process, that results from benefits and externalities provided by landscapes and ecosystems.

Keywords: Landscape, economic value, ecosystem services, benefits.

\section{Introdução}

A importância da paisagem, dos seus ecossistemas e dos seus serviços pode expressar-se através do valor ecológico, sociocultural e económico. O valor ecológico transmite o estado de saúde de um sistema e é medido por indicadores ecológicos tais como a diversidade e integridade. Este tipo de indicadores são os mais estudados e difundidos, muito devido aos avanços e contributos da ecologia da paisagem enquanto ciência e disciplina. Os indicadores socioculturais medem a importância, ou o valor dado pelas pessoas relativamente a determinados padrões e funções da paisagem, traduzindo as preferências sociais e o grau com que isso se relaciona com os serviços de ecossistemas. O valor económico, por sua vez, desdobra-se em dois tipos de valores, o valor de uso e valor de não uso. A soma dos dois quando associados a um determinado recurso ou paisagem, resulta no valor económico total $^{1}$ (Millennium Ecosystem Services, 2003 e 2005, Turner et al., 2002). O valor de uso pode ser direto ou indireto, sendo que o primeiro se refere ao valor atribuível aos recursos naturais tangíveis de consumo direto, provenientes de ecossistemas ou paisagens específicas, tais como a água, a madeira, o peixe, o petróleo ou outros. Incluem também outros recursos não tangíveis tais como aqueles relacionados com aspetos de recreio, de lazer e cénicos revelados por preferências. Os usos indiretos relacionam-se com os serviços prestados pela natureza, tal como a despoluição do ar ou a depuração da água, a prevenção da erosão e a polinização de áreas ou parcelas agrícolas.

O valor de não uso pode ainda dividir-se em quatro categorias. O valor de opção ou 'Option value' refere-se ao valor atribuível a um futuro uso expectável de um determinado recurso e poderá ser traduzido pela disposição a pagar para garantir que esse recurso se encontra disponível para uso no futuro. O valor 'Quasi-option’² é o valor atribuível ao direito de preservação das opções de uso, dadas as expectativas de conhecimento futuro, ainda não existentes num determinado momento, acerca desse recurso. $\mathrm{O}$ valor bequest 'Bequest

\footnotetext{
${ }^{1}$ Total economic Value (TEV).

${ }^{2} \mathrm{O}$ autor refere ainda que este valor pode ser considerado também um valor especulativo pelo qual o investidor pode estar disposto a pagar para preservar e de alguma forma atrasar a exploração desse recurso decorrente de um novo quadro de informação. Coggins e Ramezani (1998), referem ainda que este valor corresponde à disposição a pagar pelo direito em atrasar uma decisão.
} 
value' é o valor decorrente do pleno conhecimento de que determinado recurso, é por opção, preservado para as gerações futuras. Por fim o valor de existência ou 'existence value', é o valor atribuível ao facto de conscientemente se conhecer que um recurso simplesmente existe não havendo qualquer interesse numa utilização direta do mesmo por parte de quem está disposto a pagar por ele (Van Dyke, 2008).

\section{Preço, custo e valor em contextos de ausência de mercado}

Num contexto de avaliação económica importa distinguir as diferenças entre 'valor', 'custo' e 'preço', uma vez que correspondem a conceitos diferentes e que muitas vezes são confundidos. O 'preço' corresponde ao montante económico decorrente de um determinado ato de transação acordado entre partes. $O$ 'custo' é determinado a partir da decomposição dos encargos que terão que ser suportados para a produção de um determinado objeto. O 'valor' por sua vez corresponde a uma estimativa que informa as partes interessadas na transação de um bem e que se desdobra em múltiplos significados, tendo em conta a finalidade de uma avaliação. Na verdade, o preço de um bem raramente coincide com o valor que lhe pode ser atribuído, uma vez que o próprio conceito de valor possibilita um conjunto de interpretações mais ou menos objetivas e que poderão ser expressas em diferentes escalas sejam estas ordinais ou cardinais. A escala cardinal e especificamente a monetária é apenas uma das possíveis. No caso dos bens com mercado, ou seja, para os quais se conhecem preços de transação, pode a noção de valor ser de mais fácil tradução económica, uma vez que a ela se pode associar um conjunto de informação acerca de transações similares, que se poderão tomar como referência ${ }^{3}$ para transações futuras, e que se pode designar genericamente como 'valor de mercado'4. No que respeita aos bens sem mercado, ou seja, aqueles que não são transacionáveis e para os quais não se

\footnotetext{
${ }^{3}$ Mesmo tendo em conta a definição clássica de 'Valor de Mercado', este não nos informa de forma perfeita acerca do valor associado a um imóvel, uma vez que o mercado é por si imperfeito (Amaral, 2011). A imperfeição decorre da indissociabilidade das duas componentes distintas, mas inseparáveis da propriedade imobiliária, entendidas como o solo ou o suporte físico e as benfeitorias feitas sobre este, ou eventualmente que se poderão fazer e a que corresponderá a capacidade edificatória, agrícola, florestal, extrativa ou outra que seja geradora de renda.

${ }^{4}$ Corresponde ao preço provável, numa determinada data, em dinheiro ou termos equivalentes precisamente especificados, pelo qual os direitos específicos da propriedade devem ser transacionados numa situação de mercado competitivo, sob todas as condições de uma transação justa, na qual o vendedor e comprador atuam cautelosamente, de forma livre, informada, esclarecida e no seu próprio interesse. Appraisal Institute (http://www.appraisalinstitute.org/about/).
} 
conhecem valores em escala monetária, o problema da relação entre o 'preço' e o 'valor' coloca-se de forma mais visível. Uma vez que não existe 'mercado', o interesse em valorar bens e serviços prende-se normalmente com a necessidade de exprimir 'trade-offs' entre opções alternativas para o futuro, independentemente do conhecimento dessas relações entre ganhos e perdas ser desejado de forma consciente ou inconsciente, e como tal são, por norma, relacionados com problemas de decisão em planeamento (Van den Belt, Forgie e Farley, 2011).

Os preços não medem necessariamente as contribuições dadas pelas comodidades para o bem-estar do ser humano. Poderá tomar-se como exemplo a relação entre o preço dos diamantes e a sua contribuição para o bem-estar humano, quando comparado com o preço da água que é, por norma, bastante baixo, embora seja vital para a vida humana. As primeiras unidades de água disponíveis para consumo terão um valor incalculável. No entanto, à medida que se torna abundante e é consumida em quantidades maiores o seu preço marginal decresce, resultado de um decréscimo na importância atribuída aos usos marginais desse bem. O valor marginal de uma comodidade determina assim o seu valor de troca, ou seja, o preço de mercado ${ }^{5}$. Fisher et al., (2008) conceptualiza e explica a relação entre serviços de ecossistemas e o bem-estar humano, através de um modelo econométrico adaptado de Pearce (2007), estabelecendo relações de oferta e procura (Figura 1).

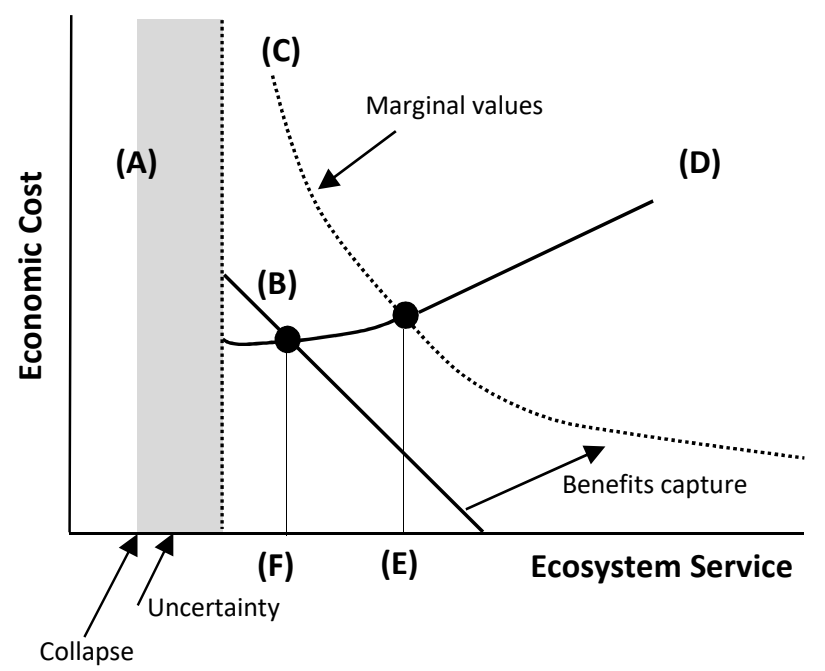

Legenda:

(A) - limite crítico das reservas de capital natural; (B) representação do comportamento típico da procura para bens e serviços com mercado; (C) - curva de procura para todos os benefícios provenientes de serviços de ecossistemas, incluindo os que não têm mercado; (D) - curva da oferta; (E) - ponto a partir do qual os custos superam os benefícios; (F) - limite inferior do intervalo onde os benefícios superam os custos.

Figura 1 - Enquadramento económico aos serviços dos ecossistemas. Adaptado de Fisher et al., (2008)

\footnotetext{
${ }^{5}$ No entanto vários autores que defendem que isso não deve ser confundido com o valor total de uma comodidade (Van den Belt, Forgie e Farley, 2011; De Groot et al., 2002; Costanza et al., 1997).
} 
Nas abcissas teremos a quantidade de serviços prestados por ecossistemas e nas ordenadas a margem de bem-estar humano, que é representado por unidade monetária, traduzindo a disposição a pagar pelo consumo de uma unidade (extra) de serviço prestado por ecossistema. A reta assinalada por B representa o comportamento típico de procura, que neste caso se refere a bens ou benefícios com mercado, e provenientes de serviços prestados por ecossistemas, tais como uma floresta na qual se extrai madeira, ou um estuário que garante uma população constante de peixe. Esse valor monetário que lhe é associado representa então a disposição a pagar pela aquisição de mais uma unidade, ou seja, representa o valor marginal associado a esse bem. Assim, à medida que os ecossistemas vão sendo convertidos e as reservas vão diminuindo, ou seja, à medida que se caminha da direita para a esquerda no eixo das abcissas, o que se espera será um aumento do valor que os consumidores estão dispostos a pagar por mais uma unidade de determinado bem, uma vez que se caminha para uma situação de escassez.

A curva de procura para todos os benefícios provenientes de serviços de ecossistemas, incluindo os que não têm mercado é aqui representada por $\mathbf{C}$, podendo incluir benefícios tais como prevenção de cheias, proteção contra a erosão, biodiversidade, etc. Por se tratar de um conjunto de benefícios agregados provenientes de serviços que não têm mercado, é expectável que esta curva se localize acima de B. A curva de oferta é aqui representada por D e representa o custo marginal em adquirir e gerir unidades adicionais de ecossistemas (e.g. hectares de terras), tal como o valor marginal dos custos de oportunidade tendo em conta os usos alternativos possíveis. Verifica-se que acréscimos na disponibilidade ou quantidade de serviços de ecossistemas implicam maiores custos económicos, sendo importante haver uma ponderação entre ganhos e perdas futuras, mesmo ao nível económico, ou seja, uma avaliação dos trade-offs resultantes em contexto de decisão.

\subsection{A importância do valor marginal}

Apesar de haver centenas de estudos sobre avaliação económica de bens e serviços sem mercado, o conceito de marginalidade aparece apenas em alguns (Fisher, 2008 citando Turner et al., 2003 e Balmford et al., 2002). Assim, para dar sentido prático à valoração, admite-se que o valor económico é uma função de mudanças marginais. As curvas de 
procura, como foram representadas, são tipicamente curvas marginais, uma vez que informam acerca de tendências esperadas de acordo com determinados fundamentos teóricos, e que o valor de determinado serviço ou benefício dado por ecossistemas, ao longo dessa curva, independentemente da métrica ou unidade de medida, é por norma uma função de pequenas variações ao longo dessa tendência. Nos mercados tradicionais, a disposição a pagar por um determinado bem, ou seja, o seu valor de mercado, é assim estimado tendo em conta o preço que alguém se disponibiliza a pagar por uma unidade adicional desse bem, ou o preço que alguém está disposto a pagar para prevenir a perda de uma unidade. Assim a questão da escala torna-se importante, uma vez que para dar sentido prático à valoração económica, importará mais saber se a conversão de mais um hectare de floresta para um uso agrícola representa uma boa ou má decisão, do que saber o valor total de todas as florestas do planeta.

\subsection{A importância da escala}

Apesar da política ambiental operar a diferentes escalas, é na escala regional e local que as decisões tomadas têm impacto real e legitimidade reforçada, seja pela própria natureza normativa decorrente da competência na atividade de planear e definir estratégias de desenvolvimento, seja pelo facto de que é a esta escala que as leis são implementadas de facto, podendo mesmo partir da escala regional e local formas de influenciar as políticas definidas ao nível nacional (Wittmer e Gundimeda, 2011). Fisher et al., (2008) refere mesmo que não será razoável, numa análise marginal, considerar-se todas as florestas do planeta como a próxima unidade, pois atribuir um valor a todo o planeta é assumir que a próxima unidade perdida serão todas as florestas no mundo. $O$ autor sugere assim que a máxima unidade plausível de mudança se situa à escala da paisagem ${ }^{6}$, e que por razões conceptuais esta entidade não tem propriamente uma dimensão pré-definida, cabendo à própria investigação definir as fronteiras de ação.

\footnotetext{
${ }^{6}$ Também aqui será importante ter em conta que uma eficaz integração da paisagem no processo de planeamento, implica igualmente uma delimitação prática de unidades de paisagem que tenha o nível de desagregação da informação harmonizado entre limites geográficos e limites administrativos, fazendo, se possível, coincidir unidades de paisagem com unidades de gestão territorial.
} 


\subsection{A importância dos custos}

Os custos adicionais decorrentes de aquisições, compensações ou genericamente de provisionamento contínuo desses serviços ou bens deve ser incorporado no processo de decisão. Deve ser então assegurado que o benefício decorrente de um aumento desse serviço do ecossistema, e que garantirá um melhor desempenho da função ou conjunto de funções prestadas pela paisagem, supera os custos implicados, tendo por base o conceito de 'equimarginality' aplicado à economia (Fisher et al. 2008; Bielsa e Duarte 2001; Leen, 1992.)

\section{Métodos de valoração económica da paisagem}

A avaliação dos serviços prestados por ecossistemas (ES) é apenas uma forma de enfatizar a questão relacionada com os trade-offs (Braat e de Groot, 2012), ou seja, fazer ponderar, através de uma escala que pode ser ou não monetária, as consequências em termos do saldo entre ganhos e perdas de determinadas opções para o futuro. Vários autores sugerem que a avaliação monetária dos ES é apenas uma das formas possíveis de medir o grau de preferência numa escala comparável (de Groot et al., 2010; Cristie et al., 2008; Fisher et al, 2008; de Groot et al., 2002; Costanza et al., 1997). Em todos os casos, trata-se apenas de dar visibilidade7, através de uma determinada métrica, a um valor que por norma, não percetível, transportando-o para o centro de um problema de decisão.

Analisando a literatura sobre métodos de valoração económica da paisagem ou ecossistemas, verifica-se que nem sempre é possível encontrar uma sistematização das diferentes técnicas em função de objetivos pretendidos. No entanto, alguns autores propõem sistematizações. Christie et al., (2008), propõe um quadro metodológico para resumir as principais técnicas analíticas e participativas disponíveis para avaliar serviços de ecossistemas, ou seja, para medir as preferências, distinguindo entre técnicas económicas e não económicas. Van den Belt, Forgie e Farley (2011), propõem uma abordagem centrada na perceção do valor em causa, ou seja, a utilização de instrumentos de valoração pode ser condicionada pela forma como o benefício prestado pelo ecossistema é ou não percebido

\footnotetext{
${ }^{7}$ A essa visibilidade muitos autores chamam "captura" do valor, podendo posteriormente servir de base a sistemas de compensação por via de políticas fiscais diferenciadas que encorajem ou desencorajem determinados comportamentos ou ações, funcionando assim como mais um instrumento de planeamento por parte da administração.
} 
pelas pessoas e nomeadamente pelos decisores (figura 2). Para os autores a decisão acerca do tipo de valoração a usar bem como o facto de poder ou não ser expressa em termos monetários, prende-se sobretudo com o contexto específico da decisão que se quer tomar ou apoiar. Se a escala temporal for curta, os benefícios forem facilmente percetíveis e não for necessário o envolvimento da população, os métodos económicos ditos neoclássicos poderão constituir-se como um adequado ponto de partida.

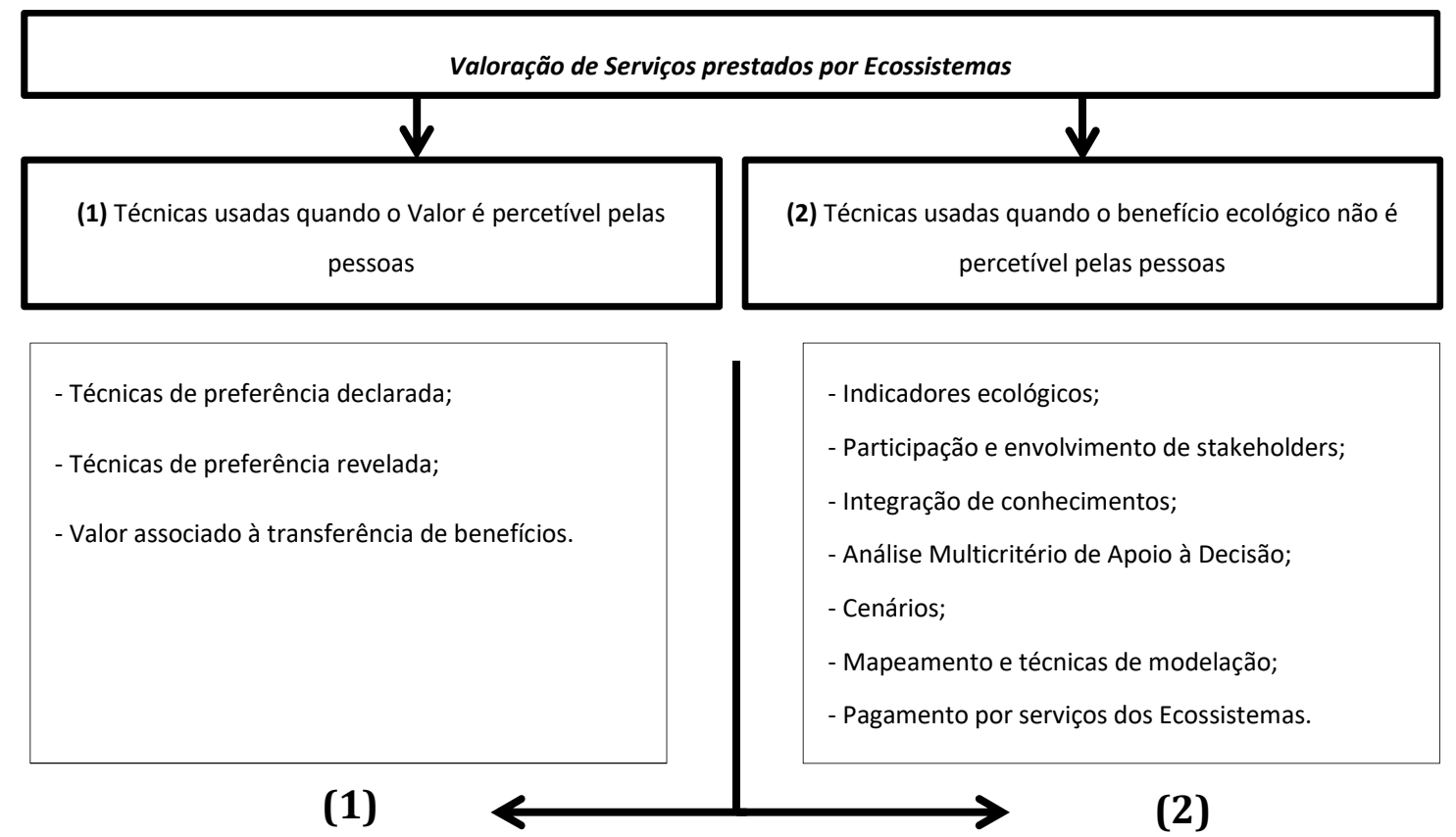

(1) Abordagem neoclássica aos princípios da economia ambiental e métodos aplicáveis quando a escala espáciotemporal é facilmente controlável e mensurável de modo objetivo;

(2) Conjunto de abordagens passíveis de utilização quando não é possível percecionar e atribuir diretamente um valor ecológico ao uma paisagem ou ecossistema. Incluem abordagens mais dinâmicas, capazes de incorporar múltiplas variáveis e pontos de vista.

Figura 2 - Valoração dos Serviços de Ecossistemas, adaptado de Van den Belt, Forgie e Farley (2011).

No entanto a abordagem neoclássica, baseada em princípios de economia ambiental, tornase bastante limitada quando o objetivo se prende com questões ligadas com a sustentabilidade a uma escala global e quando simultaneamente os valores ou benefícios em causa não são percetíveis pela população. Assim, se existem implicações a larga escala, com uma escala temporal grande, envolvendo grande número de incertezas e de pontos de vista decorrentes de um elevado número de stakeholders, torna-se necessária uma abordagem mais dinâmica capaz de incorporar múltiplos atributos e variáveis, não sendo líquido que um valor monetário seja efetivamente útil para um problema de decisão (Van den Belt, Forgie e Farley, 2011; Fisher et al., 2008). 
A abordagem neoclássica propõe métodos ditos como de preferência declarada e de preferência revelada. Os primeiros simulam condições de mercado através de técnicas de inquirição suportadas em cenários hipotéticos e em que o entrevistador cria artificialmente uma oferta a um determinado preço de certa quantidade de bens e serviços. Os entrevistados estabelecem as condições de procura através da sua disposição em aceitar (WTA) ou rejeitar as propostas. Os segundos fazem inferências a partir do comportamento observável dos mercados, obtendo valores económicos para bens e serviços prestados por paisagens ou ecossistemas específicos.

\subsection{Métodos de Preferência Declarada}

Birol et al., (2006) refere como principais técnicas a 'avaliação contingente' ou 'contingent valuation' e 'métodos de escolha discreta' ou 'choice experiments'. Van den Belt, Forgie e Farley, (2011) incluem ainda neste grupo a 'análise de preferências' ou 'conjoint analysis', através de técnicas como 'choice ranking' e 'contingent rating'. A avaliação contingente é a técnica mais frequente e difundida, funcionando melhor quando o bem ou serviço a valorar se encontra próximo de bens ou serviços com mercado. Assenta na construção de cenários cuidadosamente construídos pelo pesquisador, sobre os quais os entrevistados são questionados acerca das suas preferências. Pretende-se simular situações de mercado em que o comportamento dos participantes é observado, nomeadamente quanto à sua disposição a pagar ou 'willingness to pay' (WTP), ou ainda quanto à sua disposição a aceitar ou 'willingness to accept' (WTA) determinado valor monetário para uma mudança na quantidade ou qualidade do bem, serviço ou recurso em causa numa paisagem ou ecossistema. A média retirada da amostra é então extrapolada para a população para obter o valor global (Mitchell e Carson, 1989).

Este método é capaz de considerar valores de não uso de um recurso, bem como acomodar mudanças ambientais que ainda não ocorreram, sendo bastante importante no caso das avaliações ex-ante, através de cenários hipotéticos para determinadas medidas ou políticas com implicação direta no funcionamento da paisagem. Apesar do ser bastante frequente, apontam-se algumas fragilidades, nomeadamente: (i) a validade e fiabilidade dos montantes gerados e em especial os valores de não uso, uma vez que, dada em primeira mão alguma importância à questão monetária através da WTP, esta não requer pagamentos reais; (ii) a possíveis enviesamentos na geração e construção dos cenários; (iii) por ser sujeito a enviesamentos diversos quer por parte dos participantes quer por parte dos entrevistadores Birol et al. (2006), Kahneman e Knetsch (1992). 
Outra questão relevante prende-se com a diferença geralmente obtida entre os valores de WTP e WTA, verificando-se que as estimativas baseadas nas primeiras são por norma, menores que as estimadas nas segundas, sendo uma possível explicação, o facto das pessoas verem de forma mais negativa uma possível perda num ES do que o acréscimo equivalente nesses mesmos serviços Van den Belt, Forgie e Farley (2011).

Outras técnicas de preferência declarada incluem 'métodos de escolha discreta' ou 'choice experiment methods' (CEMs) e 'análise de preferências' ou 'conjoint analysis'. Os primeiros envolvem escolhas específicas entre diferentes níveis do mesmo atributo pelos participantes e os segundos pretendem que os participantes respondam atribuindo determinado 'ranking' ou 'rating' a diversas opções com preços pré-determinados. A principal diferença entre CEMs e a avaliação contingente, é que os primeiros permitem que os entrevistados expressem a sua WTP relativamente a um quadro de trade-offs de acordo com diferentes opções perante uma determinada mudança ambiental. Constituem-se como processos de construção de informação altamente estruturada, que depende de tarefas cuidadosamente concebidas, e cujo objetivo é revelar fatores que podem influenciar uma escolha. São definidos atributos para um determinado recurso e os níveis desses atributos são considerados em cenários que expressam uma gestão sustentável e não sustentável do mesmo. Um atributo que pode ser utilizado para descrever a qualidade das águas costeiras, será a qualidade de águas balneares. Os níveis deste atributo poderão ser 'alta', 'média' e 'baixa' qualidade. Um dos atributos será monetário o que possibilita estimar a WTP. Os perfis do recurso em causa são construídos partindo então desses atributos e de níveis de atributos obtidos através de processos específicos (e.g. 'experimental design theory' e 'statistical design theory'), combinando níveis de atributos em diferentes hipóteses que, após seleção de dois ou três perfis de alternativas, são apresentados como cenários aos entrevistados, expressando-se estes de acordo com as suas preferências (Birol et al., 2006).

Existem alguns fatores que são apontados como vantagens dos CEM relativamente à avaliação contingente. O primeiro é que, mesmo tendo em conta os valores marginais de bens e serviços de ecossistemas e paisagens específicas, os entrevistados são, por norma, mais colaborativos com a escolha de cenários do que uma abordagem diretamente assente em WTP, pois é um processo mais informativo e que consegue gerar múltiplas escolhas. Outro fator importante assenta na redução do risco de enviesamento a diferentes níveis, tais como ao nível estratégico por parte da equipa de investigação, mas também ao que Van den Belt, Forgie e Farley (2011) e Birol et al., (2006) designam como "yea-saying", ou risco de desinteresse em determinado ponto do processo. Tal como a Avaliação Contingente, os CEM podem ser utilizados para qualquer recurso ambiental inclusive para estimar valores de 
não uso. Apresenta-se ainda como uma técnica capaz de estimar o valor de atributos específicos de um recurso, para além do valor do recurso como um todo.

\subsection{Métodos de preferência revelada}

De acordo com Van den Belt, Forgie e Farley (2011), estes métodos incluem técnicas de 'custos de viagem' ou 'Travel Costs', 'comportamentos preventivos' ou 'Avertive Behaviour' e 'preços hedónicos' ou 'Hedonic Princing' (HPM). Birol et al., (2006) referem como importantes os métodos de preços hedónicos e os custos de viagem, referindo depois como menos frequentes outros como 'método de custo de substituição' (Replacement Cost Method), Comportamentos Preventivos, Production function approach, Net factor income, Cost-of-illness (COI) method e Market prices Method.

O HPM é baseado nas características da teoria do valor (Birol et al., 2006 citando Lancaster, 1966). Esta teoria diz que qualquer bem pode ser descrito como um conjunto de características e níveis que estas podem ter, sendo o preço desse bem dependente dessas características e do seu respetivo nível (Lancaster, 1966). O método HPM foi desenvolvido por Griliches (1971) para estimar o valor na mudança de qualidade de bens de consumo. É comumente aplicado para se perceber variações no preço de casas, em função do valor atribuído a recursos ambientais (Votsis, 2014; Donovan e Butry, 2011; Mayor et al., 2009; Waltert e Schläpfer, 2007; Troy e Grove, 2007; Kong et al., 2006; Morancho, 2003). O preço de uma casa vai refletir as suas características mais importantes, sejam estas o numero de quartos, o numero de casas de banho, a área, a localização e centralidade, a segurança, o prestígio, mas também os recursos ambientais disponíveis, como a qualidade do ar, os níveis de poluição sonora e visual, quantidade e qualidade da água, etc.

Assenta no pressuposto de que para a formação do preço final existe um preço implícito, não visível, que se poderá atribuir a determinadas características ou variáveis, ou seja, que existe um WTP marginal que está implícito e que representa uma valoração individual para um acréscimo de mais uma unidade de determinado recurso ambiental, muitas vezes não tangível, e que pode ser identificado estatisticamente. No entanto, serviços como controlo de cheias, melhorias na qualidade das águas, provisionamento de habitats para espécies, biodiversidade, são serviços que se encontram longe da perceção de benefício individual para o consumidor e, por isso, tornam-se difíceis de capturar utilizando os HPM (Boyer e Polasky, 2004). 
O método dos custos de viagem (TCM) é uma das mais antigas técnicas de valoração para atribuir um valor económico a áreas de recreio, fazendo uso das despesas de viagem como 'proxy'. Foi primeiramente proposto por Hotelling (1931) e posteriormente desenvolvido por Clawson (1959) e Clawson e Knetsch (1966). O TCM é usado para estimar valores de uso associados a paisagens ou ecossistemas tais como florestas, áreas costeiras, parques, praias entre outras que sejam usadas para fins recreativos e para os quais existe uma procura real de atividades de recreio, tais como caça, pesca, observação de aves, caminhadas, etc. A premissa de base é que o tempo e as despesas de viagem possam representar o preço ou WTP para ter acesso a essa paisagem. A WTP pode assim ser estimada tendo por base o número de viagens que são feitas a diferentes custos de viagem, o que, de acordo com Birol et al., (2006), equivale à disposição a pagar das pessoas por um bem de mercado baseado na quantidade de procura a diferentes preços. A TCM possibilita ainda uma variedade de modelos, permitindo inferir a várias escalas, desde modelos TCM para sítios específicos a uma escala local, até modelos regionais e mais generalistas que incorporam índices de qualidade e locais substitutos. Existem, contudo, algumas fragilidades nestes modelos, relacionadas com: (i) a capacidade de medir e definir os custos de oportunidade do tempo despendido na viagem; (ii) a difícil adequação de estratégias de amostragem; (iii) ao facto de a análise ser sempre limitada a uma situação existente e, como tal, à semelhança da HPM, não conseguir capturar os valores de não uso associados a recursos ambientais (Van den Belt, Forgie e Farley, 2011; Birol et al., 2006). Por outro lado, tende a expressar sempre um baixo WTP fornecendo um limite mínimo. Os TCM incluem ainda um número variado de modelos, tais como: (i) the individual travel cost demand model, (ii) the zonal travel cost method, (iii) the hedonic travel cost method, and (iv) the random utility model.

O método do 'custo de substituição' valoriza partindo do custo de reposição de situações em que ocorreram danos, particularmente de fatores ambientais, assumindo que esses custos são estimados partindo dos benefícios de comportamentos preventivos. Assume-se assim, neste método, que todos os danos são mensuráveis e que o valor de determinada característica ambiental danificada não é superior ao seu custo de substituição. Assume igualmente que não existem benefícios secundários que surjam com as despesas na proteção desse bem ambiental, sendo particularmente adequado quando existe um certo 'standard' que tem de ser atingido, tal como por exemplo o nível de qualidade de água (Birol et al., 2006 citando Markandya et al., 2002).

O método dos 'comportamentos preventivos', parte do comportamento de proprietários e da resposta que dão quando ocorre a degradação de um determinado bem ambiental. Quando existe um declínio acentuado de determinado bem ambiental, com poder de 
impacto direto na saúde humana, como por exemplo a contaminação da água, esperam-se determinados comportamentos preventivos para mitigar os impactos, tais como a compra de água engarrafada, redução na frequência do seu uso, ou a adoção de medidas comportamentais mais subtis, como ferver água antes do seu consumo. Os custos associados com essas mudanças de comportamento podem ser usados para estimar o valor da perda em ES. No entanto este método apresenta muitas limitações, incluindo a incapacidade de considerar mais do que um comportamento preventivo simultaneamente. Apresenta igualmente a limitação de não considerar todos os custos relacionados com a poluição, o que leva a que a estimativa seja por norma subvalorizada (Van den Belt, Forgie e Farley, 2011; Birol et al., 2006).

O 'net factor income' é um método que estima mudanças numa curva de oferta de determinado produto, subtraindo o custo de outros inputs na produção, partindo da totalidade de receitas. Assim, considerando, por exemplo, o benefício económico do melhoramento da qualidade da água, poderia ser possível medir o incremento na produtividade agrícola, ou a poupança que as autoridades têm no tratamento de águas para consumo e, com isso, obter uma estimativa económica que poderá informar acerca do valor associado a um bem ou serviço ambiental.

Outra abordagem possivel é o 'cost-of-illness method' (COI), em que o benefício associado à redução da poluição é medido pela estimativa de possíveis poupanças em despesas diretamente relacionadas com a saúde, tais como valores e pagamentos de despesa hospitalar ou em medicamentos, ou ainda considerando os custos de oportunidade tais como a perda de receita/dividendos associada com a doença. Existem, contudo, limitações que esta abordagem não considera, como eventuais comportamentos preventivos que as pessoas poderão ter para se protegerem, bem como pela sua própria natureza, ser um método pouco eficaz para valorar uma paisagem ou ecossistema.

Por último, o Método dos Preços de Mercado é usado para valorar o custo-benefício associado a mudanças na quantidade e qualidade de bens ambientais que são trocados em perfeitas condições de funcionamento do mercado. Não são por isso métodos utilizados para valorar paisagens ou ES, mas sim bens ou produtos derivados com um mercado associado, tais como a madeira e não a floresta, ou a água e não o rio (Birol et al., 2006). Verificam-se, contudo, casos em que parte do valor a que alguns produtos se apresentam no mercado se poder explicar precisamente serem provenientes de paisagens perfeitamente delimitadas, constituindo-se como exemplo alguns produtos alimentares de origem controlada e de regiões demarcadas (vinho, azeite, fruta, etc.). 


\subsection{Métodos de transferência de benefícios}

Estes são métodos de valoração económica baseados em estudos já existentes, de avaliação económica a bens e serviços sem mercado, referentes a outras áreas, sendo posteriormente aplicados à área de interesse. Van den Belt, Forgie e Farley, (2011), citando Brouwer et al., (2003), referem que em contextos de decisão, estes estudos baseados em conhecimento existente estão a ganhar cada vez mais aceitação, comparativamente a estudos baseados em investigação empírica, como os atrás apresentados. Uma razão para tal tendência é sugerida por Bateman et al., (2000), e prende-se com os elevados custos, quer em termos monetários quer em termos de tempo de decisão, referindo ainda que, apesar de poder ser usado em vários processos de decisão política, tem vindo a ser amplamente testado e usado para a estimação de benefícios percecionados provenientes de bens públicos ligados com sistemas naturais, centrando-se inicialmente apenas nos valores de uso, mas atualmente também nos valores de não uso e valores de opção. Os dois principais tipos de valoração por transferência de benefícios são a 'transferência de valor' ou 'value transfer' e 'transferência de função' ou 'function transfer' (Van den Belt, Forgie e Farley, 2011). Dentro dos primeiros Bateman et al., (2000), subdivide ainda em 'transferência simples ou desajustada de valores' e 'transferência ajustada de valores'.

A abordagem feita na Transferência Simples de Valores apresenta-se como uma abordagem simplificada e consiste em transferir os benefícios obtidos num sítio para outro, aplicando a unidade de valor estimada na fonte, também designado por "study-site", para o local onde se pretende estimar, ou "policy-site". Poderão ocorrer transferências de diferentes naturezas, sendo os mais comuns valores associados a médias ou medianas. Por sua vez, esses valores poderão referir-se a uma variedade de unidades de consumo, tais como atividades de recreio por período de tempo, ou área de um determinado recurso ambiental sujeita a medidas de conservação. Esta abordagem simples é a mais amplamente utilizada dentro dos métodos de transferência de benefícios, nomeadamente em estimativas de cálculo para locais recreativos nos Estados Unidos da América (Bateman et al., 2000). Existem, contudo, alguns aspetos críticos referidos pelo autor tais como: (i) diferenças relevantes nas características socioeconómicas em populações; (ii) diferenças físicas relevantes nos locais de origem e a estimar ('study site' e 'policy site'); (iii) diferenças nas condições de mercado; (iv) diferenças entre as propostas provisionais entre sítios, ou seja, na sua natureza e nas quantidades. 
A transferência ajustada de valores pode ser desenvolvida através de três estratégias distintas: (i) Expert jugdement; (ii) Re-analysis; (iii) Meta-analysis (Bateman et al., 2000). A estratégia Expert Judgement foi uma abordagem muito utilizada por analistas políticos nas décadas de 1970 e 1980, para uma diversidade de usos tanto na Europa como nos EUA, constituindo-se como exemplos a estimação de valores diários para usos recreativos com a água (Walsh et al., 1992), ou ainda na análise de custo-benefício para o terceiro aeroporto de Londres relativamente à estimação do valor do ruído, (Bateman et al., 2000, citando Willis and Garrod, 1994). No entanto, se para certos casos este tipo de ajustamento é aceitável, particularmente quando os benefícios são previsivelmente maiores do que os custos, já para outros são necessárias abordagens mais objetivas e ajustadas. A estratégia 'Re-analysis', ou a reanálise de amostras existentes, consiste na procura de dados existentes em sítios ou fontes observáveis (e.g. 'study-sites'), para definir novas sub-amostras de observações aplicáveis no sitio onde se quer replicar ('policy-site'). Os valores unitários podem ser estimados para essas sub-amostras no sentido de serem transferidas. No entanto, esta abordagem depende fortemente do tamanho da amostra inicial e pode gerar problemas quando é necessária uma subdivisão, ou estratificação de variáveis (Bateman et al., 2000).

Por fim, a estratégia 'meta analysis' consiste na abordagem estatística de resultados publicados em resumos de estudos e artigos com o propósito de integrar e aplicar resultados no 'policy-site' (Wolf, 1986). Foi desenvolvida nos últimos trinta anos e amplamente aplicada nos campos dos tratamentos médicos experimentais, psicoterapias e educação. Atualmente é uma técnica bastante utilizada e difundida. A 'meta analysis' transporta, entre outros problemas, o facto de que os estudos publicados poderem representar apenas a parte positiva de todos os estudos realizados, uma vez que os que apresentam resultados menos significativos tenderão a não ser publicados (Bateman et al., 2000). Ainda assim, a meta-análise oferece uma estrutura transparente na busca de padrões de resultados, relações e causalidades que permite importantes generalizações sem, contudo, violar conclusões mais interativas ou contingentes. Permite ainda extrair e integrar quantidades massivas de informação líquida que dificilmente seria obtida através de uma narrativa ou análise qualitativa (Bateman et al., 2000, citando Hunter et al., 1982; van den Bergh e Button, 1997; van den Bergh et al., 1997). Qualquer meta-análise envolve numa fase inicial uma leitura e seleção de bibliografia relevante no sentido de identificar bases de dados potenciais. Bateman et al., (2008), exemplifica recorrendo a 30 estudos sobre o valor recreativo de paisagens florestais com cerca de 100 benefícios estimados. Após análise detalhada, foi produzido um determinado número de variáveis independentes que se julgou 
poderem influenciar as estimativas de valor, tendo posteriormente sido aplicados métodos de regressão linear simples e múltipla de forma a obter-se um modelo explicativo para a variável dependente, que neste caso foi o WTP declarado. No final obteve-se um modelo robusto com cerca de $60 \%$ da variância explicada para esse conjunto de variáveis, tendo-se concluído que, de acordo com o modelo de regressão escolhido, havia um valor estimado obtido ${ }^{8}$ de cerca de $£ 0.60$ por pessoa por visita para um uso recreativo de paisagens florestais. Os resultados foram então usados para estimar a valoração dada a visitantes no País de Gales, tendo-se gerado um mapa ${ }^{9}$ com o valor recreativo, estimado com o auxílio de sistemas de informação geográfica e de modelos de 'chegadas previstas'.

Sintetizam-se de seguida (Tabelas 1 a 3 ) as principais vantagens, aplicabilidade e limitações de cada um dos métodos de valoração abordados anteriormente.

\begin{tabular}{|c|c|c|}
\hline PREFERÊNCIA DECLARADA & VANTAGENS & LIMITAÇÕES \\
\hline Avaliação Contingente & $\begin{array}{l}\text { Pode ser usado para medir o valor } \\
\text { sem observação direta. Mede } \\
\text { valores de não-uso. É uma técnica } \\
\text { facilmente entendida e permite } \\
\text { uma avaliação ex-ante e ex-post }\end{array}$ & $\begin{array}{l}\text { Técnica sujeita a enviesamentos de diferentes } \\
\text { origens (entrevistas, pontos de partida } \\
\text { conceptuais, não resposta ou resposta } \\
\text { desinteressada (yeah-saying) etc.). } \\
\text { Relativamente dispendiosa, quando } \\
\text { consideradas as fases de pré-teste e } \\
\text { desenvolvimento aprofundado de questionários. }\end{array}$ \\
\hline Choice Experiments & $\begin{array}{l}\text { Pode ser usada para medir o valor } \\
\text { de qualquer recurso ambiental } \\
\text { sem precisar de observação de } \\
\text { dados. Pode medir valores de não- } \\
\text { uso. Elimina os enviesamentos da } \\
\text { avaliação contingente e permite } \\
\text { avaliação ex-ante e ex-post }\end{array}$ & $\begin{array}{l}\text { Técnica de difícil compreensão, cara e } \\
\text { dependente da elaboração de questionários } \\
\text { aprofundados e de fases de pré-teste } \\
\text { (calibração). Envolve alguma controvérsia para a } \\
\text { aplicação de valores de não uso. }\end{array}$ \\
\hline $\begin{array}{l}\text { Conjoint Analysis (Choice } \\
\text { Ranking e Contingent Rating) }\end{array}$ & $\begin{array}{l}\text { Pode ser usada para medir o valor } \\
\text { de qualquer recurso ambiental } \\
\text { sem precisar de observação de } \\
\text { dados. Pode medir valores de não- } \\
\text { uso. Elimina os enviesamentos da } \\
\text { avaliação contingente e permite } \\
\text { avaliação ex-ante e ex-post. Torna- } \\
\text { se mais fácil de programar pela } \\
\text { pré-determinarão de categorias } \\
\text { (Ranking). }\end{array}$ & $\begin{array}{l}\text { Técnica de difícil compreensão, cara e } \\
\text { dependente da elaboração de questionários } \\
\text { aprofundados e de fases de pré-teste } \\
\text { (calibração), principalmente na divisão da } \\
\text { totalidade do ecossistema a avaliar em múltiplos } \\
\text { atributos (segmentação). Tal como a anterior } \\
\text { envolve alguma controvérsia para a aplicação de } \\
\text { valores de não uso. }\end{array}$ \\
\hline
\end{tabular}

Tabela 1 - Vantagens e limitações dos métodos de preferência declarada. Adaptado de Votsis (2014); Van den Belt, Forgie e Farley (2011); Donovan e Butry (2011); Mayor et al. (2009); Troy e Grove (2007); Waltert e Schläpfer (2007); Kong et al. (2006); Birol et al. (2006); Boyer e Polasky (2004); Morancho (2003); Bateman et al. (2000); Lancaster (1966); Walsh et al. (1992); Mitchell e Carson (1989).

\footnotetext{
${ }^{8}$ Através de um questionário aberto.

${ }^{9}$ Expresso em $\mathrm{f} /$ hectares respeitantes ao ano de 1990 e estimadas a partir das chegadas previstas e de uma distribuição da população em função da disponibilidade da rede viária.
} 


\begin{tabular}{lll} 
PREFERÊNCIA REVELADA & VANTAGENS & LIMITAÇÕES \\
\hline $\begin{array}{l}\text { Custos de Viagem (Travel } \\
\text { Costs) }\end{array}$ & $\begin{array}{l}\text { Método baseado na observação } \\
\text { direta de informação sobre } \\
\text { comportamentos e escolhas. } \\
\text { Custos relativamente reduzidos. }\end{array}$ & $\begin{array}{l}\text { Necessita de uma observação facilitada de } \\
\text { comportamentos. Limitada aos recursos existentes } \\
\text { in situ e à avaliação dessas situações específicas } \\
\text { (viagens). Limitada à avaliação ex-post. Problemas } \\
\text { relacionados com a amostragem. }\end{array}$ \\
\hline $\begin{array}{l}\text { Comportamentos } \\
\text { Preventivos (Avertive } \\
\text { Behaviour) }\end{array}$ & $\begin{array}{l}\text { Custos relativamente baixos de } \\
\text { implementação. }\end{array}$ & $\begin{array}{l}\text { Depende de informação prestada, acarretando risco } \\
\text { de enviesamento. Limitada à avaliação ex-post. } \\
\text { Incapacidade de considerar mais do que um } \\
\text { comportamento preventivo em simultâneo. Não } \\
\text { considera todos os custos relacionados com a } \\
\text { poluição, o que leva a uma subestimação do valor. }\end{array}$ \\
\hline
\end{tabular}

Preços Hedónicos (Hedonic
Princing)

Preços Hedónicos (Hedonic
Princing)

Técnica baseada em informação produzida e observada proveniente de comportamentos e escolhas reais.
Dificuldade em detetar pequenos efeitos ambientais no preço de propriedades. A conexão entre preços implícitos e escala de valores é tecnicamente complexa e por vezes empiricamente impercetível. Limitada à avaliação ex-post.

\section{Método de Custo de Substituição (Replacement Cost Method)}

Método baseado na observação direta de informação sobre comportamentos e escolhas. Custos relativamente reduzidos. Permite apurar valores mínimos de WTP.
Necessita de observação facilitada de comportamentos e gastos preventivos. As estimativas não capturam as perdas totais decorrentes da degradação ambiental. Necessita de estabelecer pontos de partida teoricamente fortes e consensuais para obter resultados fiáveis. Limitada à situação corrente. Limitada à avaliação ex-post. Não mede valores de não uso.

Valores de WTP subestimados. Limitada a avaliação ex-post. Não mede valores de não uso. de empresas que utilizam a água como input. Ancorada fortemente à teoria microeconómica. Custos reduzidos.

$\begin{array}{ll}\text { Production function } & \text { Com base em dados observáveis } \\ \text { approach } & \text { de empresas que utilizam a água } \\ \text { como input. Ancorada fortemente } \\ \text { à teoria microeconómica. Custos } \\ \text { reduzidos. }\end{array}$

Net factor income Permite avaliação ex-ante de
reservas de água, partindo de fatores externos (rentabilidade agrícola, etc.). $\begin{array}{cl}\text { Cost-of-illness (COI) method } & \begin{array}{l}\text { Custos relativamente baixos de } \\ \text { implementação. }\end{array}\end{array}$

\begin{abstract}
Abordagem tradicionalmente limitada a determinados temas específicos ligados com a qualidade e quantidade de recursos hidrológicos.
\end{abstract}

Omite a inutilidade associada à doença. Subestima valores de WTP. Limitada a avaliação da situação atual. Apenas útil em avaliação ex-post.
Market prices Method

Baseia-se em dados observáveis de escolhas reais em mercados ou outras trocas negociadas.
Não fornece valores totais (incluindo valores de não-uso). Limitado a uma avaliação da situação atual. Potencia distorções para valores de mercado quando sujeita a enviesamentos de informação.

Tabela 2 - Vantagens e limitações dos métodos de preferência revelada. Adaptado de Votsis (2014); Van den Belt, Forgie e Farley (2011); Donovan e Butry (2011); Mayor et al. (2009); Troy e Grove (2007); Waltert e Schläpfer (2007); Kong et al. (2006); Birol et al. (2006); Boyer e Polasky (2004); Morancho (2003); Bateman et al. (2000); Lancaster (1966); Walsh et al. (1992); Mitchell e Carson

(1989). 


\begin{tabular}{|c|c|c|}
\hline TRANSFERÊNCIA DE BENEFÍCIOS & VANTAGENS & LIMITAÇÕES \\
\hline Transferência Simples & $\begin{array}{l}\text { Amplamente utilizada. Método baseado } \\
\text { em conhecimento existente. Permite } \\
\text { avaliação ex-ante e ex-post. Reduzidos } \\
\text { custos de implementação. Rápida } \\
\text { implementação. }\end{array}$ & $\begin{array}{l}\text { Risco de enviesamento, uma vez que } \\
\text { não considera diferenças relevantes } \\
\text { nas características socioeconómicas } \\
\text { em populações, ou diferenças físicas } \\
\text { relevantes nos locais de origem e a } \\
\text { estimar (study site e policy site), ou } \\
\text { ainda diferenças nas condições de } \\
\text { mercado, etc. }\end{array}$ \\
\hline $\begin{array}{l}\text { Transferência ajustada de valores } \\
\text { (Expert jugdement) }\end{array}$ & $\begin{array}{l}\text { Amplamente utilizada e com diferentes } \\
\text { aplicações. Aceitável particularmente } \\
\text { quando os benefícios são previsivelmente } \\
\text { maiores do que os custos. Rápida } \\
\text { implementação. }\end{array}$ & $\begin{array}{l}\text { Risco de enviesamentos associados a } \\
\text { determinadas visões particulares ou } \\
\text { setoriais. Menos aceite quando os } \\
\text { benefícios são menores do que os } \\
\text { custos }\end{array}$ \\
\hline $\begin{array}{l}\text { Transferência ajustada de valores } \\
\text { (Re-analysis) }\end{array}$ & $\begin{array}{l}\text { Amplamente utilizada e com diferentes } \\
\text { aplicações. Rápida implementação. }\end{array}$ & $\begin{array}{l}\text { Depende em grande parte do } \\
\text { tamanho da amostra inicial podendo } \\
\text { gerar problemas quando é necessária } \\
\text { uma subdivisão, ou estratificação de } \\
\text { variáveis }\end{array}$ \\
\hline $\begin{array}{l}\text { Transferência ajustada de valores } \\
\text { (Meta-analysis) }\end{array}$ & $\begin{array}{l}\text { Baseado em conhecimento existente. } \\
\text { Estrutura transparente na busca de } \\
\text { padrões de resultados. Permite } \\
\text { importantes generalizações sem violar } \\
\text { conclusões mais interativas ou } \\
\text { contingentes. Permitem ainda extrair e } \\
\text { integrar quantidades massivas de } \\
\text { informação liquida. Permite articular com } \\
\text { outras técnicas como a avaliação } \\
\text { contingente. Permite avaliação ex-ante e } \\
\text { ex-post. Rápida implementação. }\end{array}$ & $\begin{array}{l}\text { Risco de enviesamento, uma vez que } \\
\text { os estudos publicados poderem } \\
\text { representar apenas a parte positiva } \\
\text { de todos os estudos realizados. } \\
\text { Envolve a pesquisa e estudo de um } \\
\text { número considerável de publicações. }\end{array}$ \\
\hline
\end{tabular}

Tabela 3 - Vantagens e limitações dos métodos de Transferência de Benefícios. Adaptado de Votsis (2014); Van den Belt, Forgie e Farley (2011); Donovan e Butry (2011); Mayor et al. (2009); Troy e Grove (2007); Waltert e Schläpfer (2007); Kong et al. (2006); Birol et al. (2006); Boyer e Polasky (2004); Morancho (2003); Bateman et al. (2000); Lancaster (1966); Walsh et al. (1992); Mitchell e Carson (1989).

\section{A internalização de benefícios e externalidades não tangíveis}

Além de ser possível atribuir um valor económico a um bem proveniente de um serviço ambiental $^{10}$, a análise anterior demonstra que é também possível valorar economicamente a própria paisagem, bem como até um serviço ambiental a uma escala global. Para tal deverá isolar-se o problema de decisão e ponderar acerca da utilidade em capturar esse valor tornando-o visível, e de certa forma incluí-lo na ponderação das relações entre ganhos

\footnotetext{
${ }^{10}$ Que por sua vez é suportado por uma paisagem ou ecossistema.
} 
e perdas perante futuros alternativos, utilizando e aprofundando os métodos já descritos. No entanto, no que toca à utilidade decorrente do conhecimento acerca do valor marginal de uma externalidade, em termos do seu contributo para melhores políticas de solo e para o próprio processo de planeamento, importa atender a três aspetos: (i) perceber qual é a escala onde dominantemente operam tais processos; (ii) qual o papel da Administração, uma vez que decorre essencialmente desta a obrigação de assegurar um correto ordenamento do território de acordo com princípios de justiça e equidade; (iii) quais os serviços (ambientais, sociais, culturais) a remunerar e de que forma se procede a essa remuneração.

\subsection{0 valor marginal e a escala do processo de planeamento}

Carvalho (2012) refere que cada vez mais vão sendo instituídas restrições à utilização do solo por razões ambientais, referindo ainda que existe uma tendência para consolidar da ideia de remuneração do seu desempenho ecológico. O autor propõe mesmo que essa remuneração possa ser considerada utilizando princípios baseados nos já conhecidos mecanismos de perequação compensatória, aplicando-os entre áreas urbanas e rurais, mas também entre municípios e mesmo entre regiões e países. Aragão (2011) identifica uma sistematização de mecanismos de compensação económica para serviços de ecossistemas, elaborada por P.Gutman e S. Davidson (2008) e que prevê mecanismos financeiros ao nível local, nacional e internacional. Entende-se, no entanto, que a partir de uma determinada escala, a operacionalização de tais mecanismos (e.g. tradicionais ou inovadores) deixa de fazer sentido à luz do processo de planeamento, podendo mesmo sujeitar-se a enormes dificuldades de implementação dada a complexidade inerente aos múltiplos pontos de vista que o problema de decisão pode tomar, onde o valor marginal, enquanto forma de dar sentido prático à valoração económica, pode deixar de fazer sentido, ou ainda, porque os mecanismos de harmonização de estratégias transnacionais em matéria de ordenamento do território, têm sido historicamente entendidos por muitos países como uma afronta à última fronteira de soberania de um Estado (Wittmer e Gundimeda, 2011; Fisher et al., 2008; Ferrão, 2004). 


\subsection{0 papel da administração pública}

A fiscalidade tem sido poucas vezes assumida como um instrumento de planeamento e ordenamento do território (Carvalho, 2012). Tal facto condiciona o pleno uso das competências atribuídas à administração, uma vez que a componente cartográfica dos planos não é por si só suficiente para assegurar a justa distribuição das mais-valias geradas (Condessa et al., 2013; Carvalho 2012; Correia P., 2001). Por outro lado, a ideia de um 'desempenho' ecológico do solo, tem ganho cada vez mais notoriedade e consensos na sociedade em geral, mas no entanto, não implica na prática nenhuma medida remuneratória para o proprietário, em função desse desempenho (Carvalho, 2012).

No entanto, em Portugal existem já figuras legais ${ }^{11}$ que permitem o enquadramento de pagamentos de serviços prestados pela natureza (Aragão, 2011). A autora considera ainda que todos estes exemplos se constituem como uma base para a aplicação do princípio do 'protetor-recebedor', por oposição aos já juridicamente consolidados princípios do 'utilizador-pagador' e 'poluidor-pagador'. Enquanto estes últimos procuram uma tradução económica de externalidades negativas associadas à utilização de ecossistemas em benefícios privados, fazendo com que esses custos sejam internalizados pelo beneficiário de forma a dissuadir comportamentos e incentivar a procura de soluções alternativas menos nocivas e onerosas para a biodiversidade, o primeiro pretende uma internalização à priori, do valor económico das externalidades positivas de tais serviços.

A remuneração eficaz desses serviços ambientais, em relação aos objetivos pretendidos, oscilaria entre um mínimo e um máximo, sendo o limite mínimo correspondente ao valor do

\footnotetext{
${ }^{11}$ A autora refere como principais marcos para uma reflexão futura acerca de um papel mais ativo por parte da administração os seguintes pontos: (i) a referência constitucional à compatibilização entre desenvolvimento, proteção do ambiente e qualidade de vida através da política fiscal (artigo 66, n.o2 h) da Constituição da Republica Portuguesa; (ii) a Lei das Finanças Locais que prevê que o regime financeiro dos municípios contribua para a preservação do ambiente e ordenamento do território e para o bem-estar social, através de descriminações positivas de municípios com área afeta a rede natura 2000 e pela concessão de isenções e benefícios fiscais a contribuintes que prossigam as suas atividades de acordo com padrões de qualidade ambiental e urbanísticas; (iii) Benefícios fiscais para o setor florestal, previstos no Artigo 77으 do Código Florestal; (iv) Medidas agro-ambientais no âmbito da Politica Agrícola Comum (PAC), nomeadamente o regulamento n.o 72/2009, de 19 de janeiro que contempla apoios financeiros específicos para atividades ou práticas agrícolas consideradas importantes para a proteção e valorização do ambiente (artigo 68, n.ô1 a) e v)); (v) o Programa europeu do Life+ que financia projetos relativos à natureza e biodiversidade, nomeadamente a conceção e aplicação de políticas com impacto nesta matéria; (vi) O fundo para a Conservação da Natureza e Biodiversidade previsto Artigo 37으 do DL n. 142/2008 de 24 de Julho, referente ao Regime Jurídico da Conservação da Natureza e Biodiversidade.
} 
investimento ativo quando as benfeitorias necessárias ou úteis tenham implicado custos pelo privado. Quando essas benfeitorias implicassem apenas um investimento passivo, ou seja, aquilo que define ser a 'abstenção do exercício de certas atividades', o valor mínimo a remunerar deveria ser estimado em função do que seria presumivelmente a rentabilidade média daquela atividade, naquele local e naquele ano e ainda assim com um limite. Os princípios descritos por Aragão (2011) assentam no facto de que a preservação da paisagem e dos serviços que esta presta acarreta custos, sendo a remuneração de tais serviços uma medida que procura maior justiça na repartição de encargos, podendo mesmo desincentivar o abandono de solos com baixo valor comercial, mas de elevado valor ecológico. Por outro lado, sugere que os avanços que se começam a sentir nesta matéria, no atual quadro jurídico português, não decorrem de boa vontade política ou de posturas individuais do legislador, mas antes do cumprimento de deveres já inscritos no ordenamento jurídico. Tal facto releva o papel da administração pública no sentido de criar e fazer implementar os instrumentos legais necessários aos operadores da biodiversidade para a internalização, não só das externalidades negativas, mas também das positivas que decorrem da sua atividade. Por último, torna-se por demais evidente que a necessidade de se mudar de uma perspetiva clássica, onde tradicionalmente o planeamento se encontra demasiado focado na afetação a dar ao solo urbano, e que se ancora no princípio de que apenas esse é gerador de maisvalias económicas.

\subsection{Que serviços remunerar?}

Quando se considera a hipótese de fazer implementar e gerir remunerações de serviços ecológicos ou funções ambientais, existem ainda questões cruciais que devem nortear a discussão. A questão que se considera mais pertinente prende-se com a identificação dos serviços a remunerar, uma vez que as externalidades positivas geradas por uma paisagem, ou ecossistema não beneficiam por igual todos os grupos de stakeholders (de Groot et al., 2010). De igual modo, existem serviços de ecossistemas que são considerados como bens públicos, com efeitos a uma escala mais abrangente, como a purificação do ar e serviços de filtragem de oxigénio, ou serviços de sequestro de carbono, e que normalmente não são considerados em processos de decisão pelos agentes ou gestores desses mesmos 
ecossistemas (de Groot et al., 2010, citando Balmford and Whitten, 2003; Powe and Willis, 2004). Os mecanismos de pagamento considerados podem ser implementados a diferentes escalas e de diferentes modos, no entanto, uma das questões-chave que deve ser aprofundada, de forma a ser possível passar do plano teórico para a prática, é a definição de indicadores que forneçam medidas concretas sobre os stocks ou ofertas desses serviços, bem como até o conhecimento sobre esses mesmos stocks, na perspetiva da gestão sustentável desse recurso. Assim, uma das questões mais importantes, será a capacidade estimada das paisagens e ecossistemas em fornecerem bens e serviços, uma vez que as relações quantitativas entre a biodiversidade e as componentes dos ecossistemas e dos processos e serviços são ainda muito pouco desenvolvidas (de Groot et al., 2010, citando ICSU et al., 2008).

\section{Conclusões}

É possível encontrar, na literatura científica, pontos comuns entre as abordagens com preocupações estritamente ecológicas ou ambientais, muito centradas na sustentabilidade dos recursos e outras que incorporam a função social desses bens e serviços. Nesse sentido, julga-se oportuno uma nova relação entre a ciência e a prática, nomeadamente no que toca à relação entre a ecologia da paisagem e a economia ambiental, para a definição de melhores políticas de solo em prole de maior equidade e justiça no processo de planeamento. Cabe ainda à administração encarar o uso da fiscalidade enquanto verdadeiro instrumento de planeamento, capaz de induzir as alterações necessárias a um novo entendimento sobre os solos que prestem serviços ambientais e sociais relevantes, de forma a que passem de passivos a ativos económicos.

No entanto a incerteza que é apontada, na literatura científica, quanto às relações ainda pouco desenvolvidas entre processos ecológicos e o fornecimento quantificável de determinados serviços e bens, sugere a adoção de prudência nas conclusões a retirar acerca de abordagens estritamente económicas e, principalmente, quando consideradas a escalas globais, onde a paisagem já não suporta a prática da gestão e o processo de decisão. 


\section{Referências bibliográficas}

ARAGÃO, Alexandra. A natureza não tem preço... mas devia. In: Estudos em Homenagem ao Professor Doutor Jorge Miranda. 2011.

ASSESSMENT, Millennium Ecosystem. Ecosystems and human well-being: wetlands and water. World resources institute, Washington, DC, 2005, 5.

BALMFORD, Andrew, et al. Economic reasons for conserving wild nature. Science, 2002, 297.5583: $950-953$.

BATEMAN, lan J., et al. Benefits transfer in theory and practice: A review and some new studies. CSERGE and School of Environmental Sciences, University of East Anglia, 2000.

BIELSA, Jorge; DUARTE, Rosa. An economic model for water allocation in North Eastern Spain. Published in: International Journal of Water Resources Development, 2001, Vol. 17, No. 3: pp. 397-408.

BIROL, Ekin; KAROUSAKIS, Katia; KOUNDOURI, Phoebe. Using economic valuation techniques to inform water resources management: A survey and critical appraisal of available techniques and an application. Science of the total environment, 2006, 365.1: 105-122.

BRAAT, Leon C.; DE GROOT, Rudolf. The ecosystem services agenda: bridging the worlds of natural science and economics, conservation and development, and public and private policy. Ecosystem Services, 2012, 1.1: 4-15.

BOYER, Tracy; POLASKY, Stephen. Valuing urban wetlands: a review of non-market valuation studies. Wetlands, 2004, 24.4: 744-755.

CARVALHO, Jorge. Renda fundiária, ordenamento e perequação. In: $8^{\circ}$ Congreso Internacional Ciudad y Territorio Virtual, Río de Janeiro, 10, 11 y 12 Octubre 2012. Centre de Política de Sòl i Valoracions, 2012.

CHRISTIE, Mike, et al. An Evaluation of Economic and Non-economic Techniques for Assessing the Importance of Biodiversity to People in Developing Countries. Defra, London, 2008.

COGGINS, Jay S.; RAMEZANI, Cyrus A. An arbitrage-free approach to quasi-option value. Journal of Environmental Economics and Management, 1998, 35.2: 103-125.

CONDESSA, Beatriz, et al. Prática de Perequação nos Planos de Pormenor: características, críticas e novas perspectivas. Encontro anual da Ad-Urbem, 2013.

CORREIA, P. Conflitos e Limitações dos Sistemas Actuais de Gestão Territorial. In: 1. o Congresso SFn de Direito do Urbanismo, Lisboa. 2001.

CORREIA, Teresa Pinto; ABREU, António Cancela; OLIVEIRA, Rosário. Identificação de Unidades de Paisagem: metodologia aplicada a Portugal Continental. Finisterra, 2001, 36.72.

COSTANZA, Robert, et al. The value of the world's ecosystem services and natural capital. nature, 1997, 387.6630: 253-260.

DE GROOT, Rudolf S., et al. Challenges in integrating the concept of ecosystem services and values in landscape planning, management and decision making. Ecological complexity, 2010, 7.3: 260-272.

DE GROOT, Rudolf. Function-analysis and valuation as a tool to assess land use conflicts in planning for sustainable, multi-functional landscapes. Landscape and urban planning, 2006, 75.3: 175-186.

DE GROOT, Rudolf S.; WILSON, Matthew A.; BOUMANS, Roelof MJ. A typology for the classification, description and valuation of ecosystem functions, goods and services. Ecological economics, 2002, 41.3: 393-408.

DEFRIES, Ruth, et al. Land use change around protected areas: management to balance human needs and ecological function. Ecological Applications, 2007, 17.4: 1031-1038.

DO AMARAL, Pedro Bingre. Análise das relações da política de solos com o sistema económico. Preparação da nova lei do solo, Documento Técnico dgotdu 5/2011.

DONOVAN, Geoffrey H.; BUTRY, David T. The effect of urban trees on the rental price of single-family homes in Portland, Oregon. Urban Forestry \& Urban Greening, 2011, 10.3: 163-168. 
FERRÃO, João. A emergência de estratégias transnacionais de ordenamento do território na União Europeia: reimaginar o espaço europeu para criar novas formas de governança territorial?. EURE (Santiago), 2004, 30.89: 43-61.

FISHER, Brendan, et al. Ecosystem services and economic theory: integration for policy-relevant research. Ecological applications, 2008, 18.8: 2050-2067.

HAINES-YOUNG, Roy; POTSCHIN, Marion. The links between biodiversity, ecosystem services and human wellbeing. Ecosystem Ecology: a new synthesis, 2010, 110-139.

KONG, Fanhua; YIN, Haiwei; NAKAGOSHI, Nobukazu. Using GIS and landscape metrics in the hedonic price modeling of the amenity value of urban green space: A case study in Jinan City, China. Landscape and urban planning, 2007, 79.3: 240-252.

LANCASTER, Kelvin J. A new approach to consumer theory. Journal of political economy, 1966, 74.2: 132-157.

LEEN, Auke R. Marginal utility: Short-cut in equilibrium and disequilibrium. 1992.

MAGALHÃES, M. Paisagem-perspectiva da arquitectura paisagista. Philosophica, 2007, 29: 103-113.

MAYOR, Karen, et al. A hedonic analysis of the value of parks and green spaces in the Dublin area. ESRI working paper, 2009.

MOINE, Alexandre. Le territoire comme un système complexe: un concept opératoire pour l'aménagement et la géographie. L'Espace géographique, 2006, 35.2: 115-132.

MITCHELL, Robert Cameron; CARSON, Richard T. Using surveys to value public goods: the contingent valuation method. Resources for the Future, 1989.

MORANCHO, Aurelia Bengochea. A hedonic valuation of urban green areas. Landscape and urban planning, 2003, 66.1: 35-41.

OLIVEIRA, Rosário; D’ABREU, Alexandre Cancela; SANTOS, José Carlos. QUE MULTIFUNCIONALIDADE?. Uma abordagem aplicada ao ordenamento e gestão da paisagem. Faro, 2008.

PEARCE, David. Do we really care about Biodiversity? Environmental and resource economics, 2007, 37.1: 313333.

RAMOS, Isabel Loupa. As dinâmicas da paisagem rural: a formulação de cenários como instrumento de apoio ao planeamento. 2008. PhD Thesis. Dissertação de Doutoramento em Engenharia do Ambiente. Instituto Superior Técnico. Universidade Técnica de Lisboa. Lisboa.

RAMOS, Isabel Loupa. Exploratory landscape scenarios' in the formulation of 'landscape quality objectives. Futures, 2010, 42.7: 682-692.

SOUSA MATOS, R. A Reinvenção da Multifuncionalidade da Paisagem em Espaço Urbano-Reflexões. 2010. PhD Thesis. Tesis doctoral. Universidad de Évora.

TROY, Austin; GROVE, J. Morgan. Property values, parks, and crime: A hedonic analysis in Baltimore, MD. Landscape and urban planning, 2008, 87.3: 233-245.

TURNER, R. Kerry, et al. Valuing nature: lessons learned and future research directions. Ecological economics, 2003, 46.3: 493-510.

VAN DEN BELT, M.; FORGIE, V.; FARLEY, J. 12.03 Valuation of Coastal Ecosystem Services. 2011.

VAN DYKE, Fred. Conservation biology: foundations, concepts, applications. Springer Science \& Business Media, 2008.

VOTSIS, Athanasios. Ecosystems and the spatial morphology of urban residential property value: a multi-scale examination in Finland. 2014.

WALSH, Richard G.; JOHNSON, Donn M.; MCKEAN, John R. Benefit transfer of outdoor recreation demand studies, 1968-1988. Water Resources Research, 1992, 28.3: 707-713. 
WALTERT, Fabian; SCHLÄPFER, Felix. The role of landscape amenities in regional development: $A$ survey of migration, regional economic and hedonic pricing studies. Working Paper, Socioeconomic Institute, University of Zurich, 2007.

WITTMER, Heidi; GUNDIMEDA, Haripriya (ed.). The economics of ecosystems and biodiversity in local and regional policy and management. Routledge, 2012.

WOLF, Fredric Marc. Meta-analysis: Quantitative methods for research synthesis. Sage, 1986. 